\title{
Compression of polystyrene and polypropylene foams for energy absorption applications: a combined mechanical and microstructural study
}

\author{
L. Andena ${ }^{1,2}$, F. Caimmi ${ }^{1}$, L. Leonardi ${ }^{1}$, M. Nacucchi ${ }^{3}$, F. De Pascalis ${ }^{3}$ \\ ${ }^{1}$ Dipartimento di Chimica, Materiali e Ingegneria Chimica "G. Natta", Politecnico di Milano, piazza Leonardo da Vinci \\ 32, 20133 Milano (Italy) \\ ${ }^{2}$ Engineering, Exercise, Environment, Equipment for Sport (E4Sport) Lab, Politecnico di Milano, piazza Leonardo da \\ Vinci 32, 20133 Milano (Italy) \\ luca.andena@polimi.it, tel: +39 022399 3289, fax:+39 022399 3280; francesco.caimmi@polimi.it , tel: +39 022399 \\ 4711; lidia.leonardi@polimi.it, tel: +390223993246 \\ ${ }^{3}$ ENEA, Italian National Agency for New Technologies, Energy and Sustainable Economic Development, Research \\ Centre of Brindisi, Strada Statale 7 Appia - km 706,00, 72100 Brindisi (Italy) \\ michele.nacucchi@enea.it, fabio.depascalis@enea.it
}

\begin{abstract}
In many applications polymeric foams (such as expanded polystyrene, EPS, or polypropylene, EPP) are used for protection from impacts. Standard design requires the foam to maximize the energy absorption, thus achieving large deformations (typically up to $25 \%$ and above in compression) while maintaining the stress level below a threshold value. In this work steam chest moulded EPS and EPP were characterized in relation to their density, microstructure and applied strain rate. Typical mechanical parameters (elastic moduli and plateau stress in compression) were compared with existing models and data in the literature. The strain rate dependence was accurately described using Nagy's phenomenological model. The mechanical behaviour of the foams was then correlated with their microstructure, as investigated using scanning electron microscopy and X-ray micro-tomography. Structural parameters were obtained using both $(2 \mathrm{D}$ and $3 \mathrm{D})$ techniques and relevant results were compared.
\end{abstract}

\section{Keywords:}

Polymeric foam

Energy absorption

Compression behaviour

Strain rate

Closed-cell microstructure

X-ray micro-tomography

\section{Introduction}

Closed cells materials such as expanded polystyrene (EPS) and polypropylene (EPP) are the favourite choice for the design of packaging or protective equipment. Due to their low production costs, lightness and energy absorbing capabilities, they are also exploited by sandwich structures used in the aircraft, naval and automotive industries. These materials are often processed from steam chest moulding of beads: this is a sintering-like technique to join preexpanded beads by exploiting steam heat and pressure. This process is practical in realising complex geometries; for example, it is the favourite one for packaging solutions. There are many variables involved which may affect the final performance of the manufactured equipment; as an example, the effect of the production parameters on the tensile behaviour of EPP bead foams was investigated by Zhai W. et al. [1]. So far the behaviour of foams has been found to be dependent on the manufacturing method, gas properties (for closed cell foams), cell geometry and obviously the constituent material [2].

Foams absorbing capability is the key reason for their use in many applications. Their ability to absorb kinetic energy whilst keeping the peak force and acceleration below a certain threshold, which would cause damage or injury, is of critical importance. These characteristics come from the peculiar stress-strain behaviour displayed by these materials (see Fig. 1). 


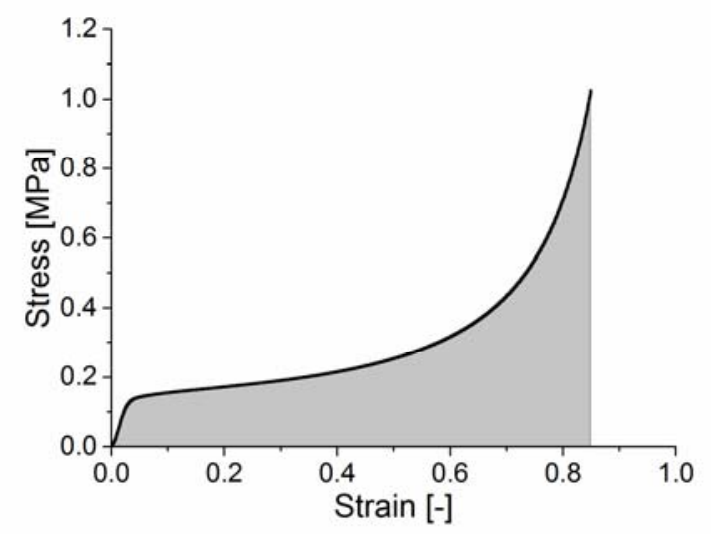

Fig.1 Typical uniaxial compression stress-strain behaviour of a closed cell foam. The grey area represents the absorbed energy per unit volume.

There are several ways of characterising the energy absorbed during single shock or multiple impacts [2]. For example, the Janssen factor $J$ relates the impact peak deceleration of the real foam to that of an "ideal" foam, able to absorb all the impact energy at constant deceleration. This factor depends on thickness, impact energy and needs many empirical data in order to be assessed. An alternative way is to evaluate the Cushion factor, which can be obtained from curves representing the absorbed energy as a function of the peak stress; this can be easily derived from existing uniaxial stress-strain data. The analysis was further improved by Rusch [3-4], whose approach lead to the construction of normalized (with respect to the foam modulus) peak stress vs. specific energy absorption curves. Although this method provides a greater generality than the use of the $J$ factor, it still relies empirically on the evaluation of the foam's response under different levels of impact energy, although some attempts at modelling the cushioning behaviour have been made by Burgess et al. [5] and Castiglioni et al. [6]. A more refined approach is given by the construction of the so-called Maiti diagrams [7], in which the energy per unit volume is plotted against the stress level (both normalized to the foam's modulus) for different foam densities. The set of points corresponding to the densification onset for each density is used to define an envelope curve that can be used to select the proper density and material for a given application. This method also needs extensive data at different strain rates in order to cover general application requirements.

In view of predicting the behaviour of foams under given conditions, with the objective of optimizing the design of energy absorption devices, various models have been developed, trying to capture the actual mechanics of the material involved. Among existing micromechanical models, the one from Gibson and Ashby [2] is commonly used to gain a basic understanding of the foam behaviour. This model is based on the assumption of a regular cell structure; it can be used to describe the experimental behaviour of irregular structure foams, but the assumption somewhat limits its predicting capabilities. Other models include those by Kelvin [10], Shulmeister [11] and Roberts-Garboczi [12]; the latter two try to overcome the limitation of an assumed regular microstructure. Yet, all these models do not allow a realistic representation of the actual foam structure and thus they can only provide limited accuracy.

An attractive way of describing the mechanical behaviour of foams is to rely on numerical modelling [8-9]. Some authors (e.g. [13-14]) used micro-computed tomography to reproduce the true foam structure with a finite element method (FEM) code, in some cases even coupled with Digital Image Correlation (DIC) [15]. However, the determination of mechanical parameters for the material composing the cell walls and struts is very difficult; its properties are likely to significantly differ from those of the bulk solid polymer. Moreover, it can be very difficult to correctly resolve the finer microstructural details. An alternative approach is to use a homogenized model able to reproduce the macroscopic behaviour of various foam types: many commercial FEM codes include potentially suitable material models. The first challenge is a thorough determination of relevant material parameters. A large amount of data is available regarding the quasi-static uniaxial compression behaviour of lightweight closed cell polymeric foams [2, 16-20]. There is a great interest in the correlation between foam physical behaviour and finite element results, but up to now FEM models of polymeric foams are still far from being accurate [8].

In view of further efforts directed at improved FEM modelling of polymeric foams, the aim of the present work is to extensively characterise and compare the mechanical behaviour (in the quasi-static regime) of EPS and EPP foams having varying densities. Particular attention is given to the strain rate dependence of the properties, which is essential for applications dealing with impacts, albeit testing was performed under quasi-static conditions. A correlation was 
sought with microstructural features of the foams identified by performing Scanning Electron Microscopy (SEM) and $\mathrm{X}$-ray micro-computed tomography $(\mu \mathrm{CT})$ analyses.

\section{Theoretical background}

\subsection{Gibson and Ashby model}

In the following section we review the model by Gibson and Ashby [2], which is widely employed to describe the compression behaviour of low density cellular solids; in the present work it was used for data analysis.

Closed cells foams (see Fig. 1) are expected to show an initial linear elastic behaviour, when cells edges shrink or bend and cells membranes stretch, contributing to the axial cell-wall stiffness. The fluid contained within the cells also partially contributes to the stiffness. This regime is followed by a stress plateau corresponding to progressive cells collapse: the plateau extends up to large values of strain. This region is responsible for the large energy absorbing capability of these materials. When the cells are crushed, opposing cell walls come in contact and further strain compresses the solid itself, giving the final region of rapidly increasing stress (densification). After this stage the material behaves closely to the original (i.e. non expanded) solid. The quasi-static compression properties are commonly measured at strain rates below $10^{-1} \mathrm{~s}^{-1}$.

The plateau stress $\sigma_{p l}^{*}$ can be expressed as a function of the foam relative density $\frac{\rho^{*}}{\rho_{s}}$, given by the ratio of the foam density $\rho^{*}$ over the solid polymer density $\rho_{S}$, by the following equation:

$$
\frac{\sigma_{p l}^{*}}{\sigma_{y}} \approx 0.3\left(\Phi \frac{\rho^{*}}{\rho_{s}}\right)^{3 / 2}+0.4(1-\Phi) \frac{\rho^{*}}{\rho_{s}}+\frac{p_{0}-p_{a t}}{\sigma_{y}}
$$

where $\sigma_{y}$ is the solid material yield stress; $\Phi$ the volume fraction of solid in the edges; $p_{0}$ is the fluid pressure inside the cells and $p_{a t}$ is the atmospheric pressure.

The dependence of the elastic modulus $E^{*}$ on relative density $\frac{\rho^{*}}{\rho_{s}}$ is expected to follow Eq. (2):

$$
\frac{E^{*}}{E_{S}} \approx \Phi^{2}\left(\frac{\rho^{*}}{\rho_{s}}\right)^{2}+(1-\Phi) \frac{\rho^{*}}{\rho_{s}}+\left(\frac{p_{0}}{E_{S}}\right) \frac{\left(1-2 v^{*}\right)}{\left(1-^{*} / \rho_{s}\right)}
$$

$E_{s}$ stands for the bulk material elastic modulus, $v^{*}$ is the foam Poisson's coefficient.

For low-density fluids and moderately low strain rates, the third, pressure dependent term in both Eq. (1) and (2) can be neglected; this was the case in the present work.

\subsection{Strain-rate dependence}

A linear dependance of $\sigma_{p l}^{*}$ on the logarithm of strain rate, $\log (\dot{\varepsilon})$, is often observed. An increasing behaviour was reported at high strain rates for relative densities above 0.1 [2,16]; Ouellet et al. showed that these effects become more pronounced between 500 and $1000 \mathrm{~s}^{-1}$ for EPS and HDPE foams [21]. This behaviour was modeled by Gibson and Ashby as an additive stress contribution proportional to the applied strain rate and was correlated to micro-inertia effects expected in case of small cell edge length $(\mu \mathrm{m})$, cell walls thickness and high cells fluid viscosity. According to this, microcellular foams may be significantly strain-rate dependent even at relatively low strain rates.

Nagy [22] proposed an empirical power law to describe the strain rate dependence of foams, which had been successfully applied by many authors [16,23-24]:

$$
\sigma(\varepsilon)=\sigma_{0}(\varepsilon)\left(\frac{\dot{\varepsilon}}{\dot{\varepsilon}_{0}}\right)^{n(\varepsilon)}
$$

In this model, $\sigma_{0}(\varepsilon)$ represents the material stress-strain behaviour at a reference strain rate, $\dot{\varepsilon}_{0}$. According to Nagy's findings, the index $n(\varepsilon)$ is expected to linearly depend on the actual strain level.

\section{Materials and methods}

\subsection{Mechanical tests}

EPP and EPS closed-cell foams with varying nominal densities, listed in Tables 1 and 2, respectively, were tested; additional properties (elastic modulus and density) of the relevant solid material, as given by the producers, are listed, too. In the following each material will be indicated by a code including the base polymer and density: for example, 
EPP-75 stands for EPP with $75 \mathrm{~kg} / \mathrm{m}^{3}$ density. EPP steam chest moulded sheets (13 mm thick) were provided by JSP Corporation (Japan); they were cut using a band saw and a scalpel into cube-like specimens. EPS samples produced by the same technique were supplied in the form of cubic blocks by Versalis S.p.A., Italy. The cubes' final dimensions were about $13 \times 13 \times 13 \mathrm{~mm}^{3}$ and $40 \times 40 \times 40 \mathrm{~mm}^{3}$ for EPP and EPS, respectively; the exact dimensions of each sample were carefully measured.

Table 1 EPP foam densities

\begin{tabular}{|c|c|c|c|c|c|c|c|c|}
\hline & \multicolumn{7}{|c|}{ EPP $\left(\right.$ solid: $\left.E_{s}=1300 \mathrm{MPa} ; \rho_{\mathrm{s}}=950 \mathrm{~kg} / \mathrm{m}^{3}\right)$} \\
\hline $\begin{array}{c}\text { Density } \rho^{*} \\
{\left[\mathrm{~kg} / \mathrm{m}^{3}\right]}\end{array}$ & 20 & 35 & 55 & 60 & 75 & 90 & 110 & 120 \\
\hline $\begin{array}{c}\text { Relative density } \\
\rho^{* / \rho_{\mathrm{s}}[-]}\end{array}$ & 0.022 & 0.039 & 0.061 & 0.067 & 0.083 & 0.100 & 0.122 & 0.133 \\
\hline
\end{tabular}

Table 2 EPS foam densities

\begin{tabular}{|c|c|c|c|c|}
\hline & \multicolumn{3}{|c|}{ EPS (solid: $\mathrm{E}_{\mathrm{s}}=3300 \mathrm{MPa} ; \rho_{\mathrm{s}}=1050 \mathrm{~kg} / \mathrm{m}^{3}$ ) } \\
\hline $\begin{array}{c}\text { Density } \rho^{*} \\
{\left[\mathrm{~kg} / \mathrm{m}^{3}\right]}\end{array}$ & 10 & 13 & 19 & 25 \\
\hline $\begin{array}{c}\text { Relative } \\
\text { density } \rho^{* /} \\
\rho_{\mathrm{s}}^{*}[-]\end{array}$ & 0.010 & 0.013 & 0.019 & 0.025 \\
\hline
\end{tabular}

Uniaxial compression tests were run on an Instron 1185R5800 dynamometer equipped with a $10 \mathrm{kN}$ load cell, under crosshead displacement control conditions. All the tests were run up to nominal strains of $70-90 \%$ at $\mathrm{T}=23^{\circ} \mathrm{C}$ and $\mathrm{RH}=50 \%$.

Five samples per density were tested at three strain rates: $3.2 \cdot 10^{-3}, 3.2 \cdot 10^{-2}$ and $3.2 \cdot 10^{-1} \mathrm{~s}^{-1}$. Displacement data was corrected for machine compliance, which was measured running a set of tests without a foam sample between the compression plates. To verify any possible material anisotropy due to the sheets production process, EPP samples were tested both along the in-sheet plane and in the perpendicular (thickness) direction.

\subsection{Microstructural characterization}

The microstructure of EPP samples was investigated with 2D (SEM) and 3D ( $\mu$ CT) imaging techniques, looking for a correlation with the results of mechanical testing; Scanning Electron Microscopy (SEM) and X-ray micro-computed tomography were used to analyse the foam microstructure on both as-received and compressed foam samples.

For SEM imaging, specimens were immersed in liquid nitrogen and subsequently broken by bending, to limit any possible damage to the material surrounding the relevant fracture surface. They were then metallised with palladium before conducting the analysis using a Zeiss EVO 50 EP-SEM.

The $\mu \mathrm{CT}$ scan of foams was performed by a GE Phoenix Nanotom CT system equipped with a $180 \mathrm{kV} / 15 \mathrm{~W}$ nanofocus X-ray tube and a 2300 x 2300 pixel Hamamatsu flat panel detector. A molybdenum target, suitable for weak absorbing specimens, was used. The sample size allowed a geometrical magnification of 15 times and a nominal spatial resolution (voxel size) of $3.3 \mu \mathrm{m}$. The X-ray tube parameters were set as follows: accelerating voltage of $50 \mathrm{kV}$ and beam current value equal to $180 \mu \mathrm{A}$ for the foams EPP-60, EPP-90 and EPP-120; for EPP-35 samples the previous values become 40 $\mathrm{kV}$ and $220 \mu \mathrm{A}$, respectively. The detector integration time was $1.250 \mathrm{~s}$ for the uncompressed samples and $1.500 \mathrm{~s}$ for the compressed ones. Each projection was obtained by averaging three 2D X-ray images, in order to improve the signalto-noise ratio. The number of projections was 2400 , giving a total scan time of approximately three hours and half or four hours for untested and tested samples, respectively. The volume reconstruction was carried out with the proprietary application software Phoenix datos $\mid \mathrm{x} 2$ reconstruction.

The 3D visualization and analysis software Avizo 8 Fire Edition of Visualization Sciences Group (a FEI Company) was used for the image processing of the datasets. In order to characterize the cell foam features, the following processing steps were performed: de-noising of grey-scale images by median filter, segmentation by thresholding, pore separation 
and labelling by watershed algorithm, removing all the pores touching the borders of the image and, finally, calculation of cell features [25-26].

An attempt to analyse the EPP-75 foam was made by further stretching the acquisition time up to 5 hours and improving the voxel resolution; however, the quality of the reconstruction was not sufficient for the analysis. The large number of smaller cells (with respect to other densities), combined with the low contrast, prevented a successful segmentation.

\section{Results and discussion}

\subsection{Mechanical tests}

Fig. 2 shows representative uniaxial stress-strain curves for $90 \mathrm{~kg} / \mathrm{m}^{3} \mathrm{EPP}$ and $19 \mathrm{~kg} / \mathrm{m}^{3}$ EPS samples, at an intermediate strain rate. Each figure shows results from five samples, indicating a good overall repeatability, which also occurred for the other densities and strain rates.
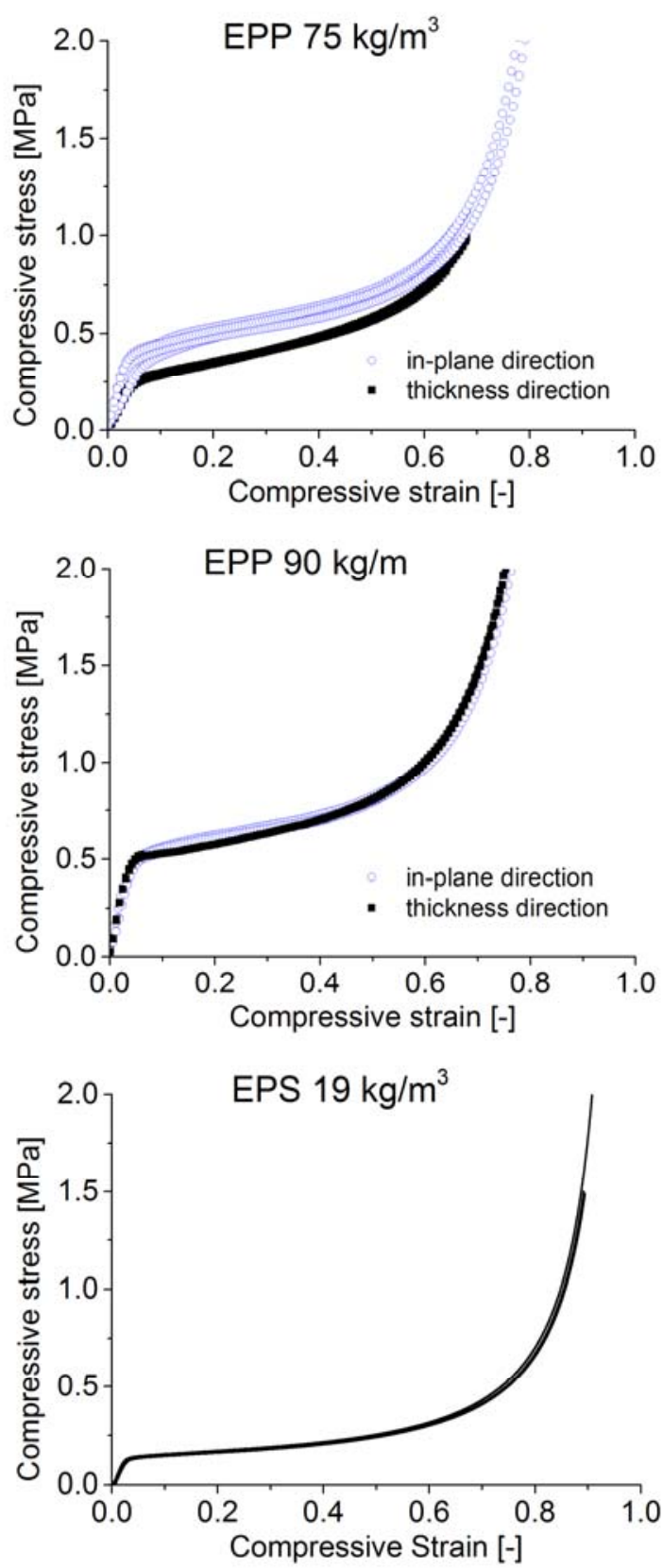

Fig.2 Uniaxial compression stress-strain behaviour of EPP-75, EPP-90 and EPS-19 at a strain rate of $3.2 \cdot 10^{-2} \mathrm{~s}^{-1}$. 
Irrespective of the loading rate the behaviour of the foams was in most cases isotropic: this is evident in Fig. 2 which shows an almost identical behaviour for EPP-90 samples having different orientation. However, we noticed a significantly anisotropic behaviour for EPP-75, as shown in Fig.2: samples tested along the sheet in-plane direction displayed a notably higher plateau stress. This anisotropy was most likely induced by the steam chest moulding process parameters and by the specific mould geometry. In the following, only data along the thickness direction will be considered.

Average curves showing the compressive behaviour at varying density for EPS and EPP foams are shown in Fig.3. The expected trend of increasing modulus and plateau stress with increasing density is observed. The energy absorption increases as well, at the price of a higher stress for a given strain. Again, EPP-75 is noteworthy as the only exception: its data cross the EPP-60 average curve, showing lower modulus and plateau stress but an earlier onset of densification. Together with anisotropy, this fact suggests that EPP-75 differs somewhat (aside from density) from the other EPPbased materials. For this reason, EPP-75 data have been highlighted in the following graphs.
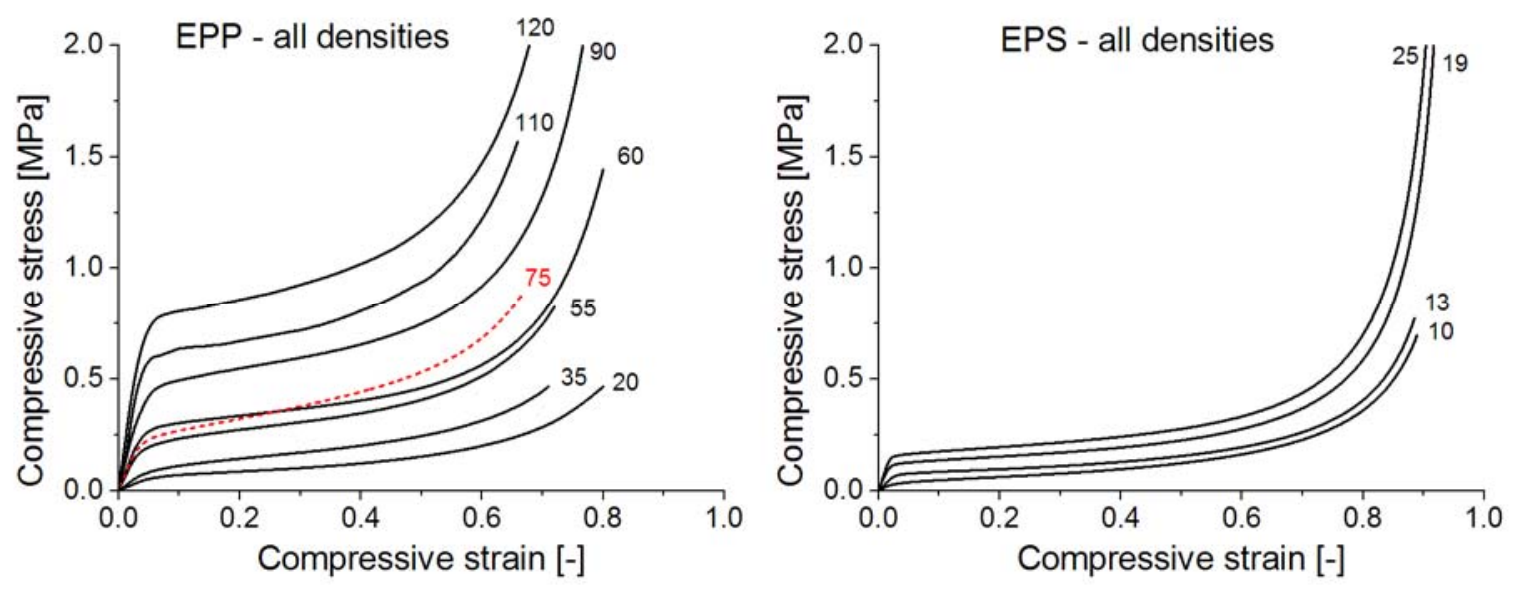

Fig.3 Effect of material density on the compression stress-strain behaviour of EPP (thickness direction) and EPS at a strain rate of $3.2 \cdot 10^{-3} \mathrm{~s}^{-1}$. Labels indicate foam density.

These materials exhibit a moderate strain rate dependency, more evident in the case of EPP; Fig.4 shows the stressstrain behaviour of both materials at varying applied strain rate for two given densities. A very limited effect was observed on the elastic modulus, well within the measured experimental scatter; a more marked influence is visible on the plateau stress level, which increases with increasing strain rate. Consequently, in the following analysis the rate dependence of the elastic modulus has been neglected and relevant measurements taken at the three rates were treated as a single dataset.
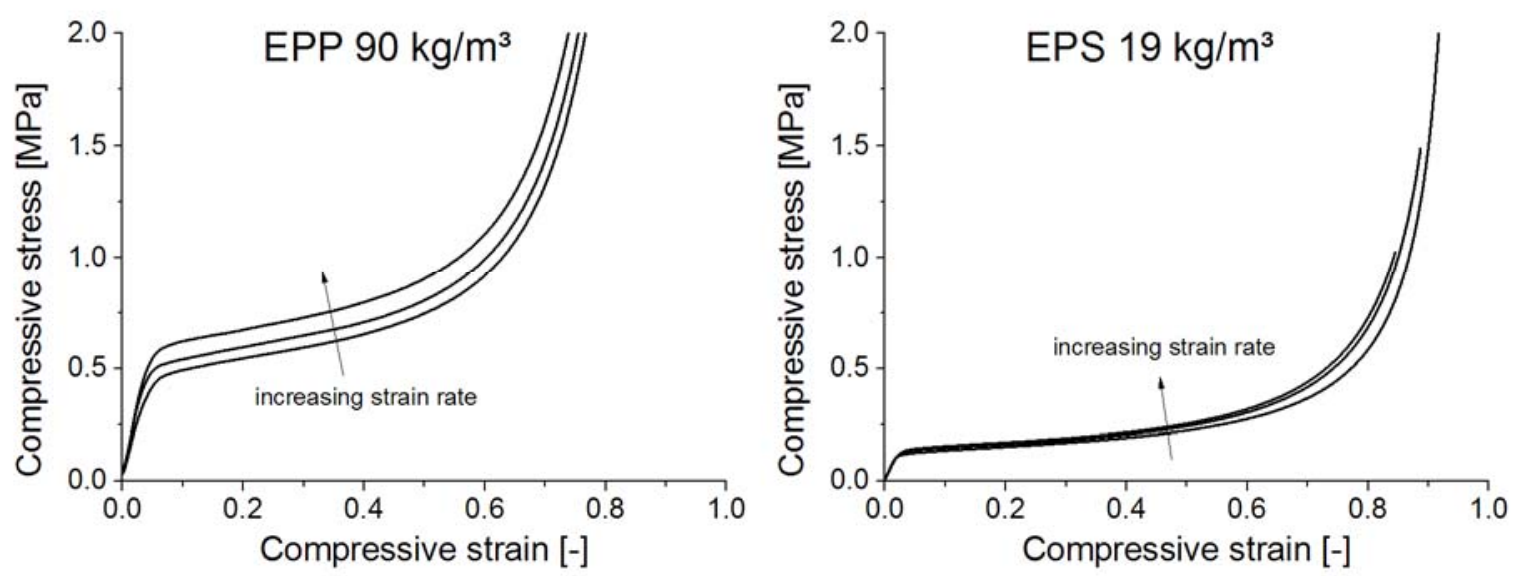

Fig.4 Effect of strain rate on the compression stress-strain behaviour of EPP-90 and EPS-19. 
Figs. 5 and 6 show the elastic modulus and plateau stress (conventionally defined as the compressive stress at $25 \%$ strain) of both foams plotted against their density. A fit to the Gibson-Ashby model, Eq. (1) and (2), was attempted using the Levenberg-Marquardt algorithm provided by OriginPro (v9.0) software; for this purpose, the strain rate dependence of both elastic modulus and yield stress was neglected. At first, elastic modulus vs. density data was fitted with the values of $E_{s}$ and $\rho_{s}$ reported in Table 1 and 2, allowing for the determination of the volume fraction of solid in the edges; $\Phi=0.985$ and $\Phi=0.921$ were found for EPP and EPS, respectively. The value for EPP is unusually high for a closed cell foam; nevertheless, these $\Phi$ were subsequently used to fit plateau stress vs. density data. In this step relevant values for the solid polymer yield stress of $\sigma_{y}=75 \mathrm{MPa}$ and $\sigma_{y}=135 \mathrm{MPa}$ were found for EPP and EPS, respectively.

Despite mild inconsistencies, the Gibson-Ashby model can well describe all data, with the only exception of EPS modulus for very low densities. The unrealistic values determined for $\Phi$ clearly indicate that the model, in its simplicity, cannot accurately represent the complex nature of the real sintered foams under investigation; yet, it still proves itself able to capture the dependence of mechanical properties on the foam density. It is interesting to observe that, while still in acceptable agreement with the overall tendency, data for EPP-75 slightly stand out from the rest and lie a bit lower than what could be expected based on the model predictions; this fact is in agreement with the aforementioned findings.
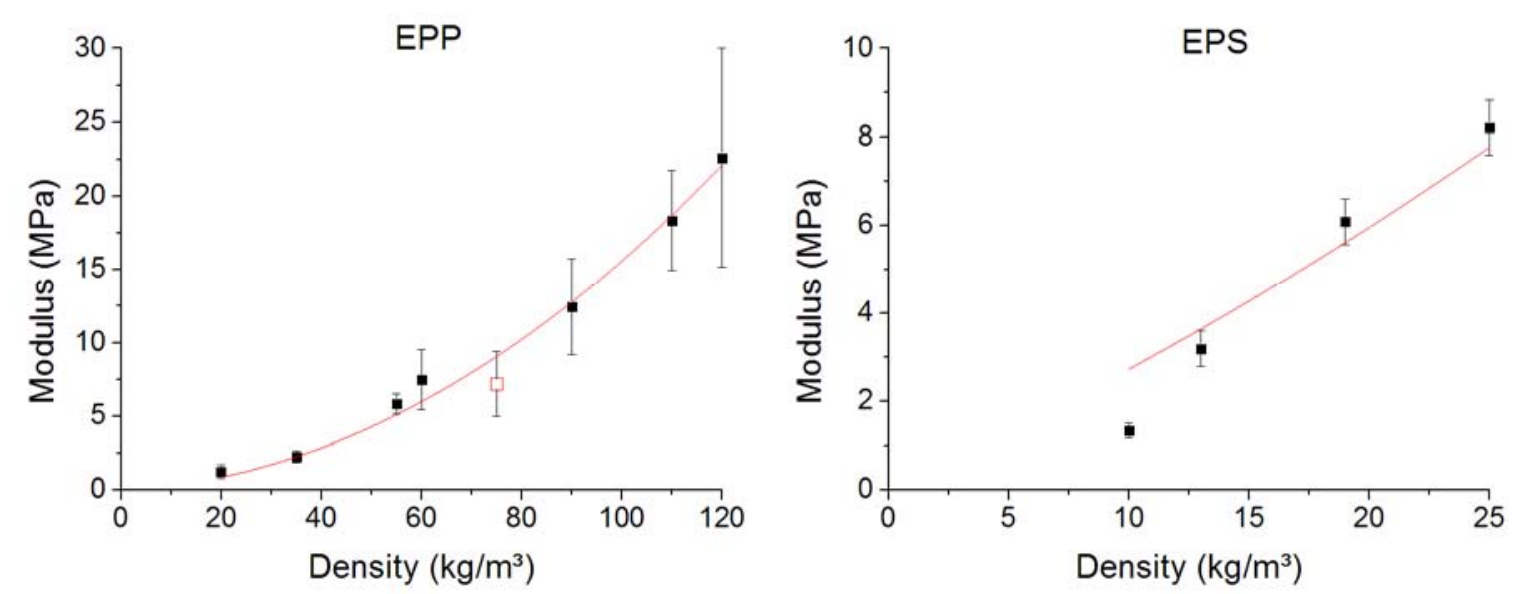

Fig.5 EPP and EPS elastic modulus data represented as a function of nominal density. Continuous lines: fit to Eq.2.
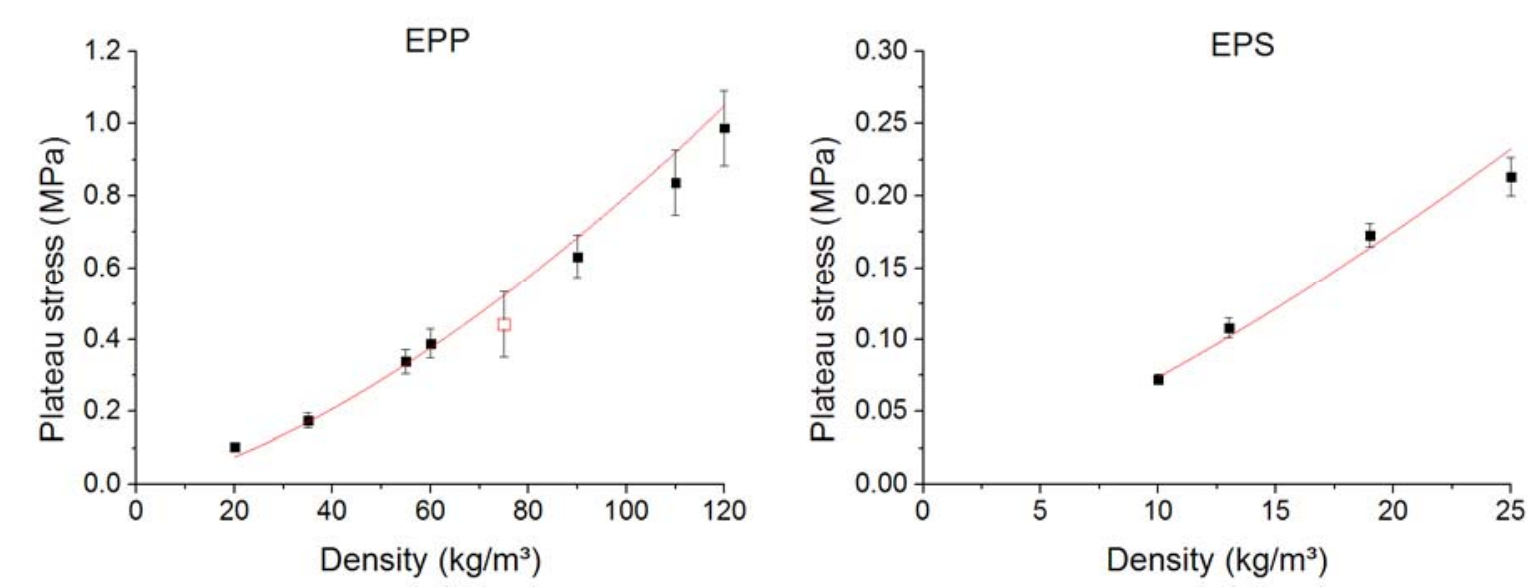

Fig.6 EPP and EPS plateau stress data (at 25\% compressive strain) represented as a function of nominal density.

Continuous lines: fit to Eq.1.

The relatively large scatter of elastic modulus prevented the identification of a clear trend with varying strain rate. Its origin might lie in the sample irregularities generated by cutting, as material is removed and incomplete cells, walls and edges are exposed on the surface which will come in contact with the testing fixtures, The presence of a slightly 
irregular free surface exerts greater influence on the behaviour at small strains, which is relevant for the determination of elastic modulus.

Concerning the plateau stress, on the other hand, a power-law dependence of $\sigma_{p l}^{*}$ versus the the applied strain rate was observed, as can be seen from the log-log plots of Fig. 7. Data are in good agreement with Nagy's empirical model represented by Eq. (3); in particular, the slope given by the index, $n(\varepsilon)$, is almost independent of density for a given material. A slightly higher slope was found for EPP-75 but this material also displays a relatively larger data scatter.
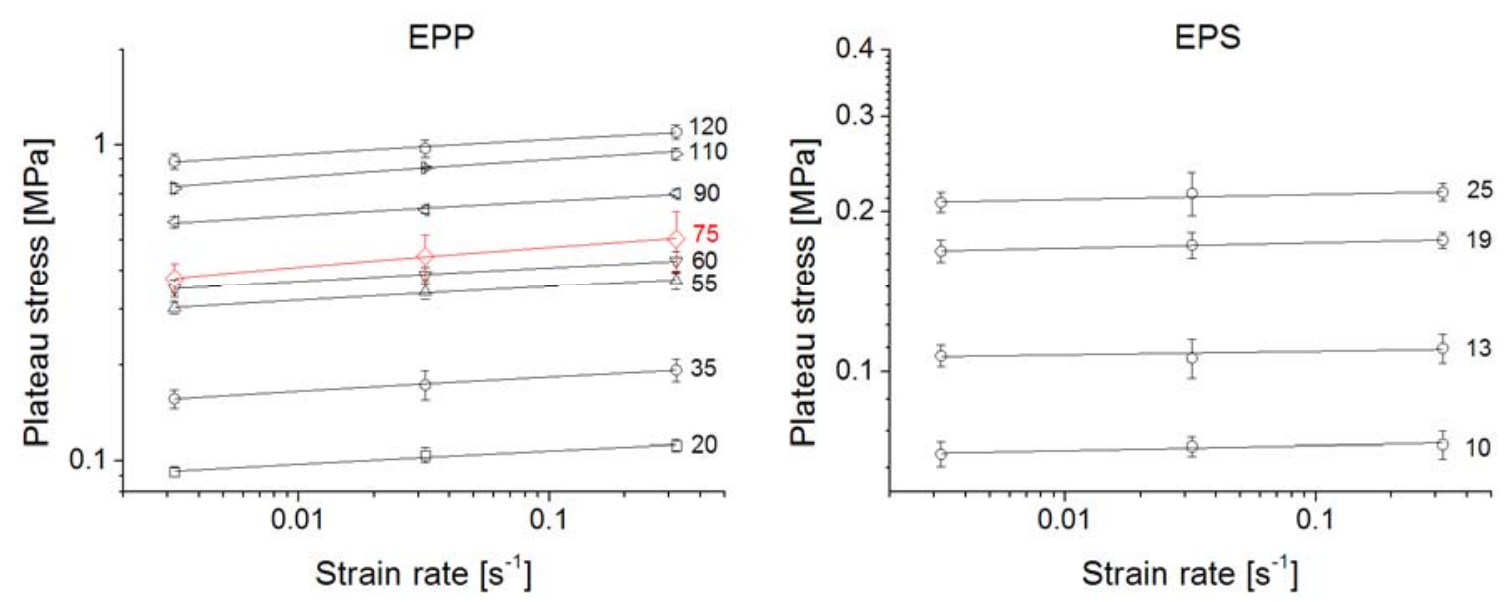

Fig.7 EPP and EPS plateau stress data (at 25\% compressive strain) represented as a function of strain rate. Continuous lines: fit to Eq.3. Labels indicate foam density.

When comparing slopes for different strain levels, no significant difference could be found between materials having different densities. As a consequence, relevant data was averaged and a single curve of $n$ versus the applied compressive strain was obtained for each material (EPP and EPS); results are shown in Fig. 8.

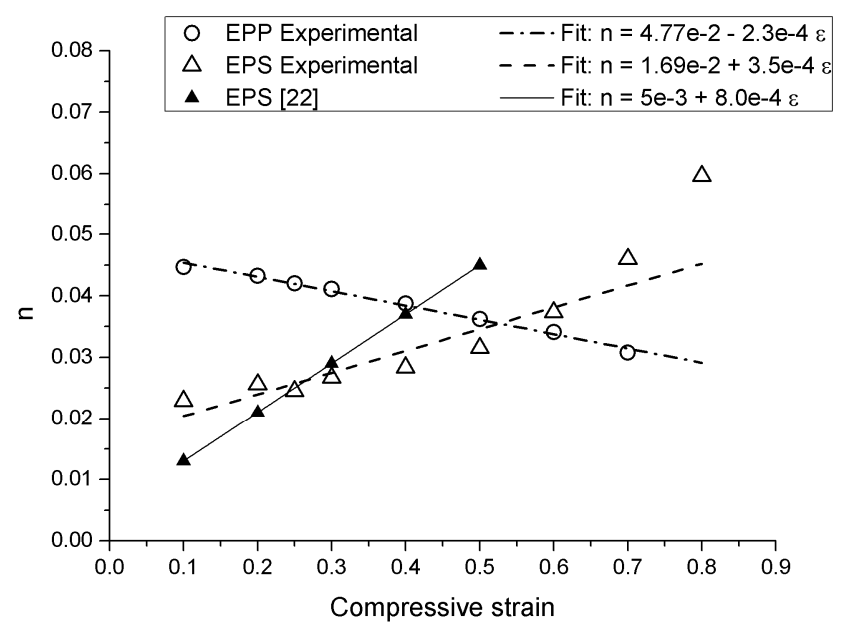

Fig.8 Dependence of the strain rate index $n$ (see Eq. 3) on the compressive strain for EPP and EPS.

Lines represent a linear fit of the data.

A linear fit of the data was attempted, in accordance with Nagy's findings. Indeed, EPP data for $n$ exhibits a linear dependence on the compressive strain, although the strain rate index is decreasing with increasing strain - at variance with results on the materials investigated by Nagy [22]. On EPS, $n$ increases with increasing strain but there is only a fair agreement between experimental data and the linear fit, with a consistent deviation for higher strains. The experimental values are in the same range as those reported in [22] for a polystyrene foam having a density of $20 \mathrm{~kg} / \mathrm{m}^{3}$; however, it must be noted that those data displayed a significantly larger degree of scatter (compare for example Fig. 7 with Fig 16. in [22]). The purely empirical nature of Nagy's model is not of help in understanding what the physical meaning of $n$ might be and what could be expected in terms of its dependency on the applied strain level; this is even more true when considering materials, such as EPS (amorphous glass) and EPP (semicrystalline with rubbery amorphous phase), which possess a markedly different structure at room temperature - most likely related to different 
deformation mechanisms active in the two polymer. As an example, polystyrene is expected to yield by crazing while in the case of polypropylene shear yielding will be the dominant mechanism under similar conditions. The latter is certainly due to a combination of inherent non-linearity in the foam constituent material as well as geometrical nonlinearity arising from the foam microstructure. For this reason, it is quite unlikely that the proposed linear trend of $n$ with $\varepsilon$ would hold for very large deformations, at which densification occurs yielding a dramatic change in the foam behavior.

To evaluate the energy absorption properties of the foams, relevant diagrams were constructed following Maiti's approach [7]; they are presented in Fig. 9. From the uniaxial stress strain curves, the absorbed energy, $W$, vs. stress curves were constructed, for all foam densities and strain rates; the energy was normalized by the foam volume, $V$. For each strain rate, the envelope of the "shoulder" points corresponding to densification defines a curve, which gives the locus of optimal energy absorption for a given material; in this way, the correct density can be chosen depending on the maximum tolerated stress level [7]. The densification points are depicted as symbols in Fig. 9, while the corresponding envelopes are shown as continuous lines. Energy and stress values were normalised by the solid material modulus to allow a comparison of the two foam types (EPP and EPS) on the same graph.

In the range of densities studied, the envelopes for both materials are reasonably approximated, on a log-log scale, by straight lines. Within the experimental scatter, there is no significant difference in the absorbed energy per unit volume at varying strain rate, the envelopes for the different strain rates being almost coincident. The corresponding slopes for the EPP foam are about 1.1, slightly lower than those for the EPS foam which lie in the range 1.3-1.4. Such values are higher than those predicted by the Ashby-Gibson model for open-cell foams whose dissipative behaviour is dominated by plasticity, which should display a slope of 1 [2]. Possible reasons for this discrepancy could be a significant contribution to dissipation of air trapped in the closed cells or a deviation from the model caused by the significant strain hardening shown by the presently studied foams.

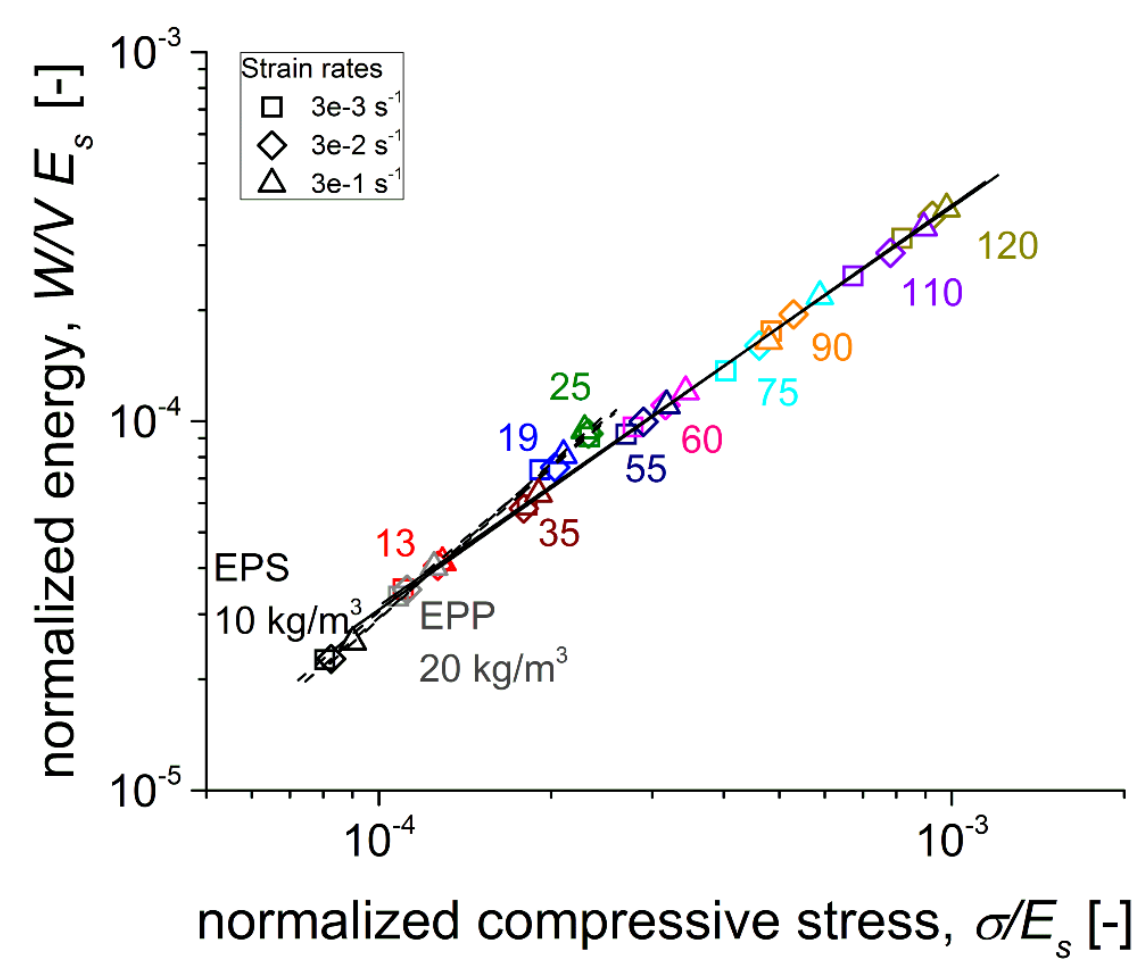

Fig. 9 Energy absorption diagram for EPS and EPP foams. Different colors correspond to different densities; see the online version of this figure. Labels below the data points refer to the EPP foams, while labels above refer to the EPS ones.

\subsection{Microstructure analysis}

In Fig. 10 (a)-(c) 40X SEM micrographs of EPP foams for selected densities are shown. The $75 \mathrm{~kg} / \mathrm{m}^{3}$ foam has a microstructure which is characterised by a large number of smaller cells if compared with that of the other foams; this is true also for the densities not shown, which are similar to the ones in Fig. 10 (a) and (c). To quantitatively evaluate this difference, the mean Feret diameter [27] and cell area distribution were measured using standard image processing tools (provided by the ImageJ software package). The results are shown in the plots in Fig. 10 (d) and (e). While both 
quantities slightly increase with increasing density, for the $75 \mathrm{~kg} / \mathrm{m}^{3}$ foam (shown in red) the distribution is shifted towards significantly smaller values.

Very similar results stand also for wall thickness, Fig. 10 (f), for which a more pronounced positive trend with increasing density can be observed; again, the EPP-75 foam clearly differs from the rest, with the lowest wall thickness among all densities. These differences in the microstructure of the $75 \mathrm{~kg} / \mathrm{m}^{3}$ EPP foam influence its mechanical behaviour, as outlined in the previous subsection.

The cell area distributions span about one order of magnitude. Such wide amplitude can be explained in part by the fact that the material is made from beads: the cells close to beads edges tend to become smaller and oblate, as it can be observed by looking at the image for the $35 \mathrm{~kg} / \mathrm{m}^{3}$ foam (Fig. 10(a)), where a boundary between two beads is clearly visible.

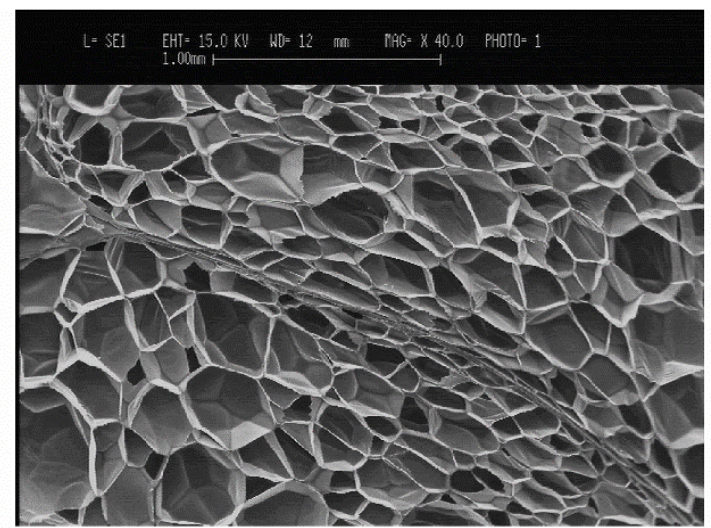

(a) $35 \mathrm{~kg} / \mathrm{m}^{3}$

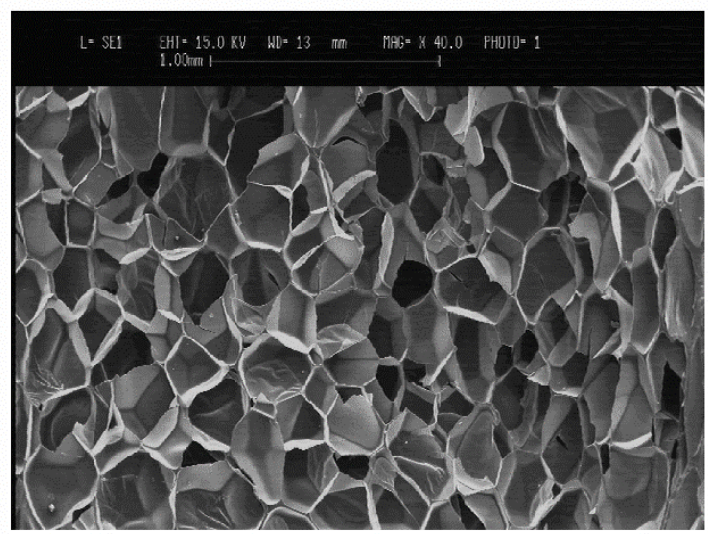

(c) $120 \mathrm{~kg} / \mathrm{m}^{3}$

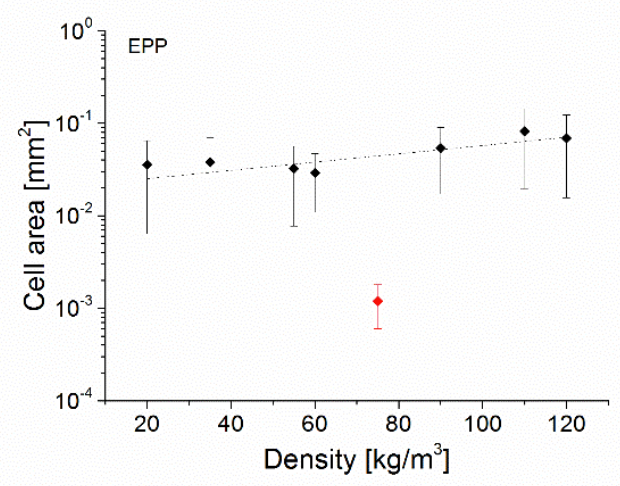

(e)

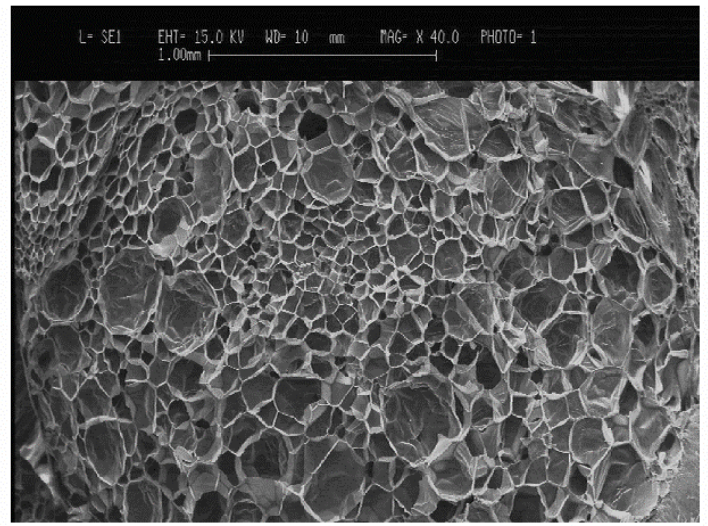

(b) $75 \mathrm{~kg} / \mathrm{m}^{3}$

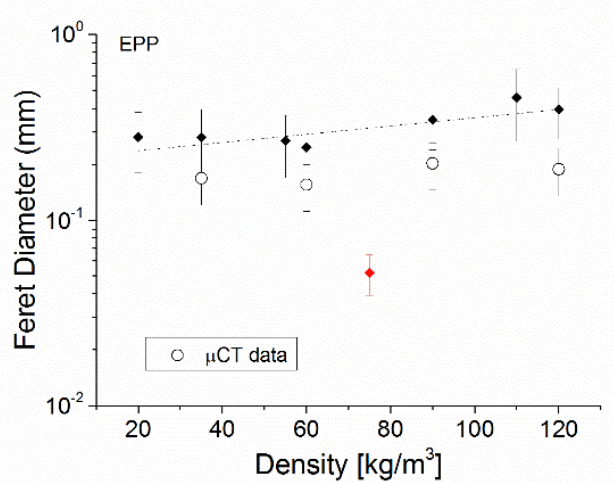

(d)

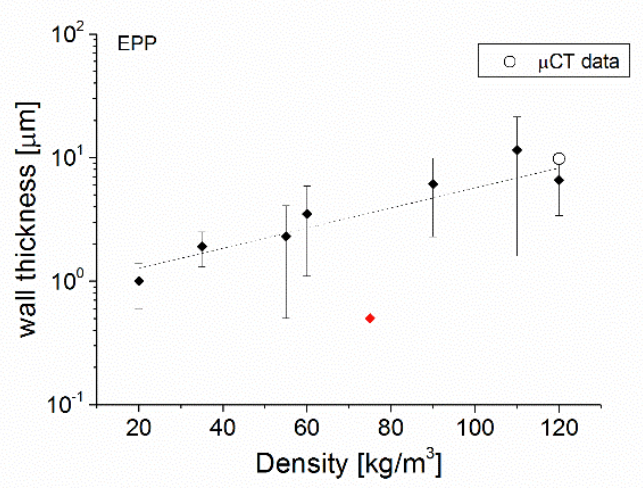

(f)

Fig. 10 EPP foam microstructure: SEM images for selected densities ((a)-(c)). Diagrams of mean Feret diameter (d), cell area (e) and wall thickness (f) distribution; dashed lines represent a linear fit of all data except for EPP-75 (in red). Error bars indicate the standard deviation of the measurements. For Feret diameter and wall thickness, available $\mu$ CT data are also included. 
A consequence of the high dispersion in the cell size population is that any quantitative evaluation of cell properties must be carefully conducted being aware that results may display very large local fluctuations. A statistical analysis is required to draw any conclusive observation and the process of examining a large number of samples with a 2D technique such as microscopy can be very time-consuming. For this reason, it is fairly obvious to turn to $3 \mathrm{D}$ techniques such as $\mu \mathrm{CT}$ analysis, which can gather data over a large cell population with a single analysis.

The volume of interest (VOI) in the $\mu \mathrm{CT}$ analysis of the untested (NT) EPP foams was a cube having an edge length of 1200 pixels, corresponding to an absolute length of $4 \mathrm{~mm}$. As later shown, this region, despite its small size, contains several thousand cells, a number of objects sufficient for a reasonable statistical analysis. Fig. 11 shows two virtual sections (slices) which cross the centre of the EPP-90 NT foam sample.

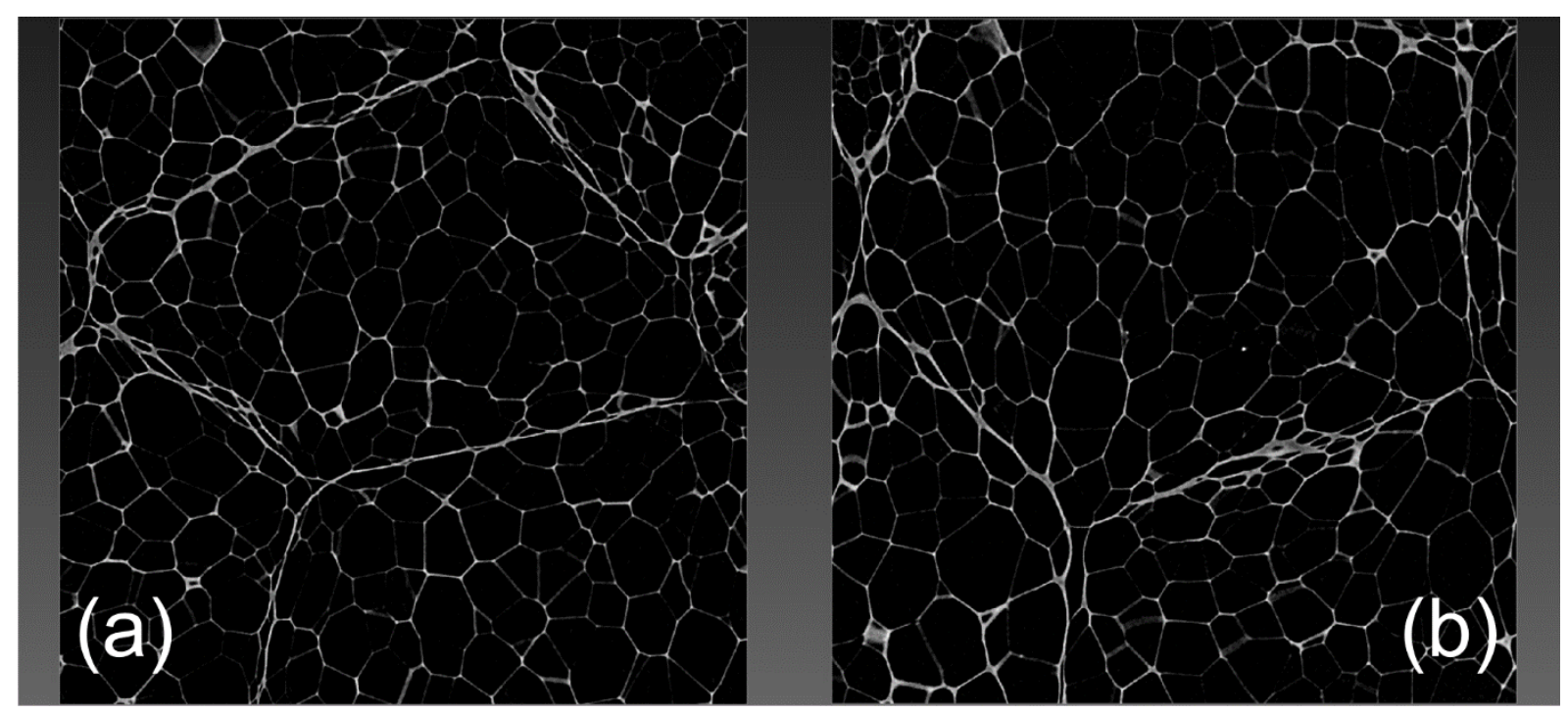

Fig. 11 Orthogonal slices of the central part of the EPP-90 NT sample. (a) virtual section along the thickness direction; (b) virtual section along the perpendicular plane (in-plane).

The same slices after the image segmentation process are shown in Fig. 12. The cells area is filled by using eight different colours to facilitate the distinction between a particle and its surrounding ones.

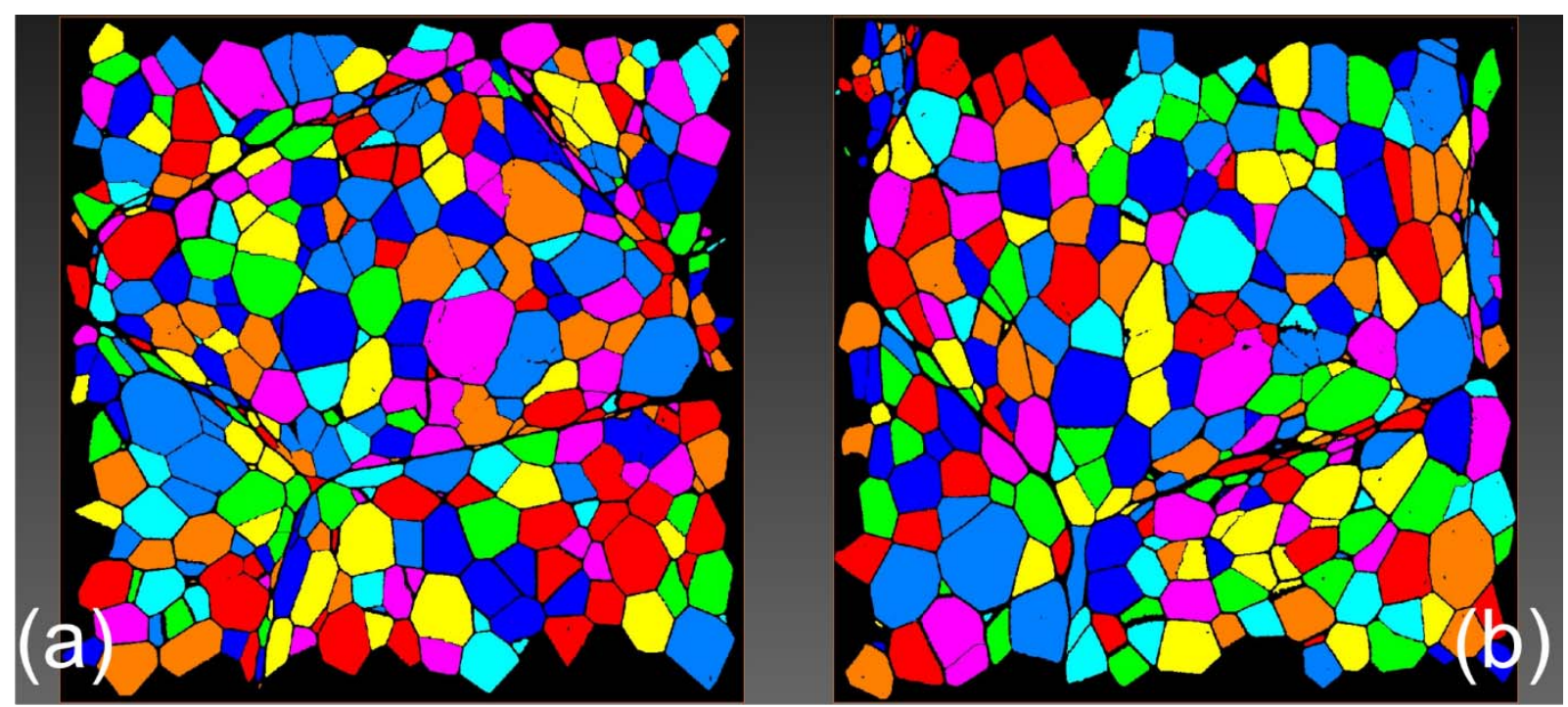

Fig. 12 The same orthogonal slices of the central part of the EPP-90 NT sample as in Fig. 11(a-b), after the segmentation process. The border cells were removed.

Note that the cells along the beads walls appear to be deformed, as seen also in the SEM images. These flattened cells were removed from the following analysis, to avoid introducing biases in the quantitative description of the overall cell shape and size distribution. A filtering based on the measure of the "sphericity" of the cells was thus applied. Sphericity is defined as the ratio of the surface areas of an equal volume sphere and that of the selected particle [28]. An ideal 
spherical particle has a sphericity of 1 while real ones will have decreasing values as their shape deviates from that of a sphere [26-28], as in the case of the flattened cells along the beads walls. A threshold value equal to 0.63 was taken for the EPP-90 NT foam, rejecting all the cells having a lower sphericity; this value was chosen as it corresponds to a sharp increase in the slope of the sphericity distribution diagram, as shown in Fig. 13 (a), thus identifying the region where the relatively few deformed cells are. The number of rejected cells was 708; the total number of analysed cells was 5449 . Figure 13 (b) shows the volume rendering of the 100 largest rejected cells; note that besides the deformed cells, the figure shows also few clusters of small cells which were not well separated by the watershed algorithm and were then excluded from the analysis. The datasets corresponding to untested foams having different density were filtered in a similar manner, with threshold value ranging from 0.58 to 0.63 and the number of rejected cells varying from a minimum of 708 up to a maximum of 1227. The percentage volume of the rejected cells with respect to the total cells volume amounts to only a few pct., ranging from 2.4\% (EPP $120 \mathrm{NT}$ ) to $6.8 \%$ (EPP $35 \mathrm{NT}$ ). These values give a slight overestimation of the total volume of voids between beads inside the foams.
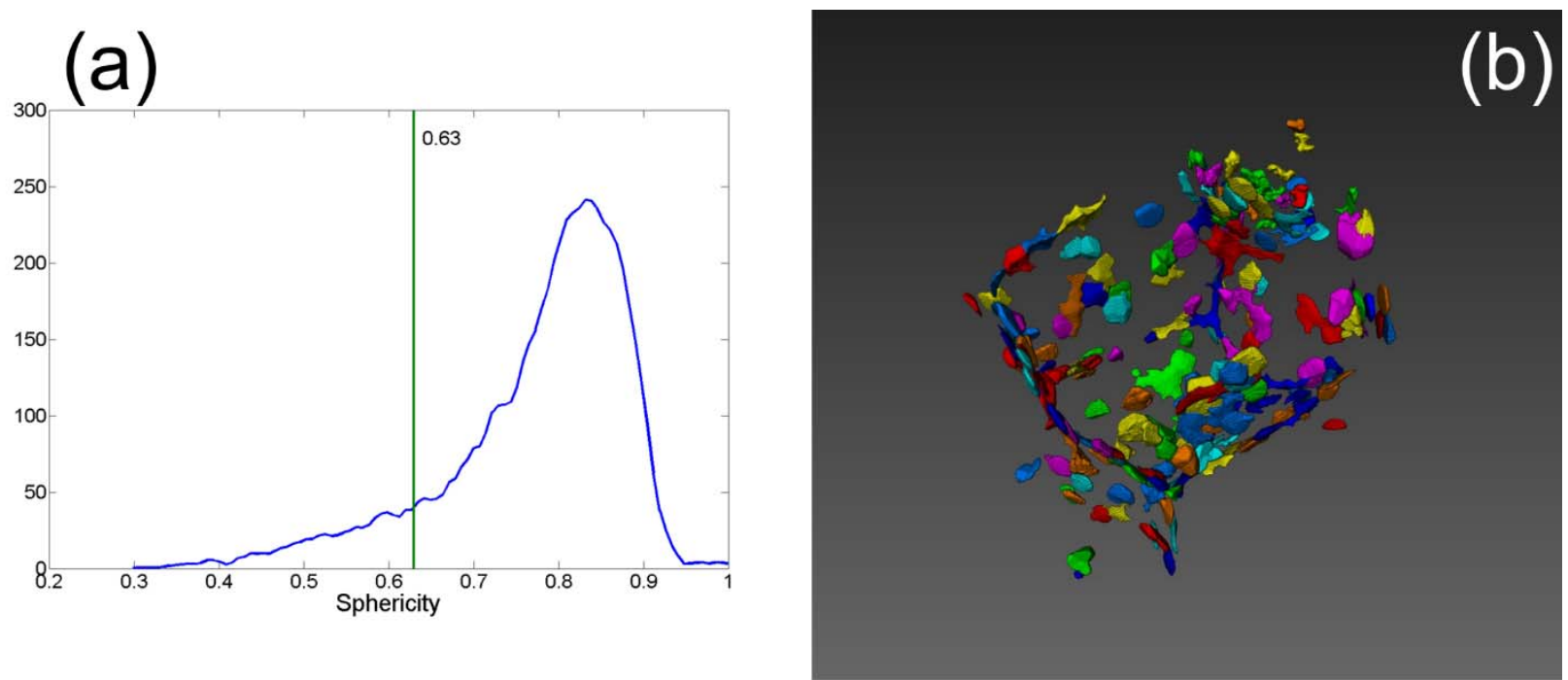

Fig. 13 (a) Sphericity distribution diagram for the EPP-90 NT sample; (b) volume rendering of the 100 largest rejected cells, after the filtering process. Note the flattened shape of the cells.

Finally, local structural features of individual cells such as volume, size, shape and anisotropy were statistically evaluated. The size was measured by computing both the Feret and the equivalent diameter, i.e. the diameter of an equal volume spherical particle. Feret diameter was evaluated to have a direct comparison with SEM data, as seen in Fig. 10(d); $\mu \mathrm{CT}$ analysis gives values which are close but consistently lower than those obtained from the 2D cut surfaces. This seemingly strange result clearly highlights the difference that exists between the analysis of many plane sections versus the entire volume. Let us consider the EPP-120 foam, where the difference is more pronounced, as an example. Had the 993 cells with a diameter less than $50 \mu \mathrm{m}$ (those corresponding to the peak near the origin in Figure 14) been eliminated, the average 3D Feret diameter would have been equal to $399 \mu \mathrm{m}$ : this value is virtually identical to that obtained by processing the 2D SEM images. The mean value of the diameter of the excluded cells was approximately $25 \mu \mathrm{m}$. The thickness of the relevant micro-tomography slices is equal to $3.3 \mathrm{um}$. Therefore, on average, one of these excluded cells "occupies" 7.5 slices. The total number of slices is 1200 . Dividing 1200 by 7.5160 slices are obtained, each $25 \mu \mathrm{m}$ thick. Now dividing 993 by 160, a result of 6.2 is obtained. This means that for a randomly cut a sample, only 6 cells with an equivalent diameter of less than 50 um can be found on average on any given two-dimensional section. An accurate survey shows that these cells are mainly located in the most chaotic area near the edges of the beads. By looking at a two-dimensional image, it is not difficult to overlook six tiny cells on more than one hundred. Yet even these 6 or 7 cells neglected in the plane view can lead to a peak near the origin in the 3D analysis, shifting the centre of the total distribution.

The sphericity was used to characterize the cell shape, as already stated. Anisotropy was determined for each cell by evaluating the momental ellipsoid of a hypothetical rigid body having the same shape; the ratio, $r$, between the minimum and maximum axes length was then calculated. A local anisotropy parameter was conveniently defined as $A=(1-r)$ : in this way, an isotropic particle has $A=0$; by contrast, for a one-dimensional fibre $A=1$ [26, 28-29]. As an example, Fig. 14 shows the equivalent diameter and the anisotropy distributions for the EPP-90 NT foam sample. 

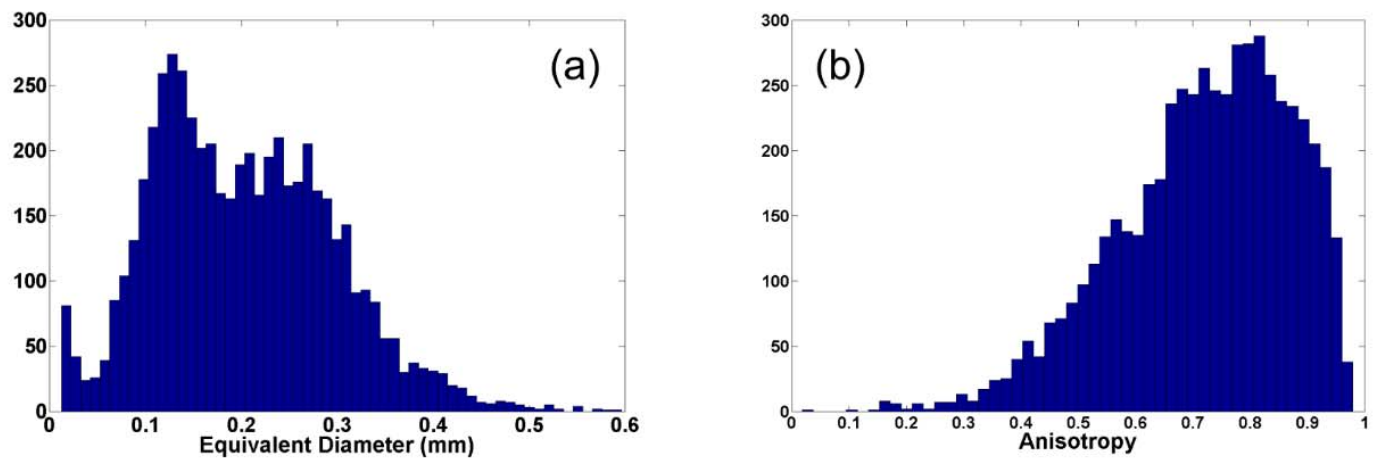

Fig. 14 Histograms of equivalent diameter (a) and anisotropy (b) distributions related to foam EPP-90 NT.

The tested samples were analysed in a similar manner. The VOI in the $\mu \mathrm{CT}$ analysis of the tested (T) EPP foams was a rectangular cuboid having a base length of 1200 voxels and a height of 2000 voxels, corresponding to an absolute size of $4.0 \mathrm{~mm} \times 4.0 \mathrm{~mm} \times 6.7 \mathrm{~mm}$. The VOI was chosen so as to exclude the top and bottom regions of foam samples, adjacent to the compression plates. Fig. 15 shows two virtual sections (parallel to the testing direction) which cross the centre of the EPP-90 T foam sample.

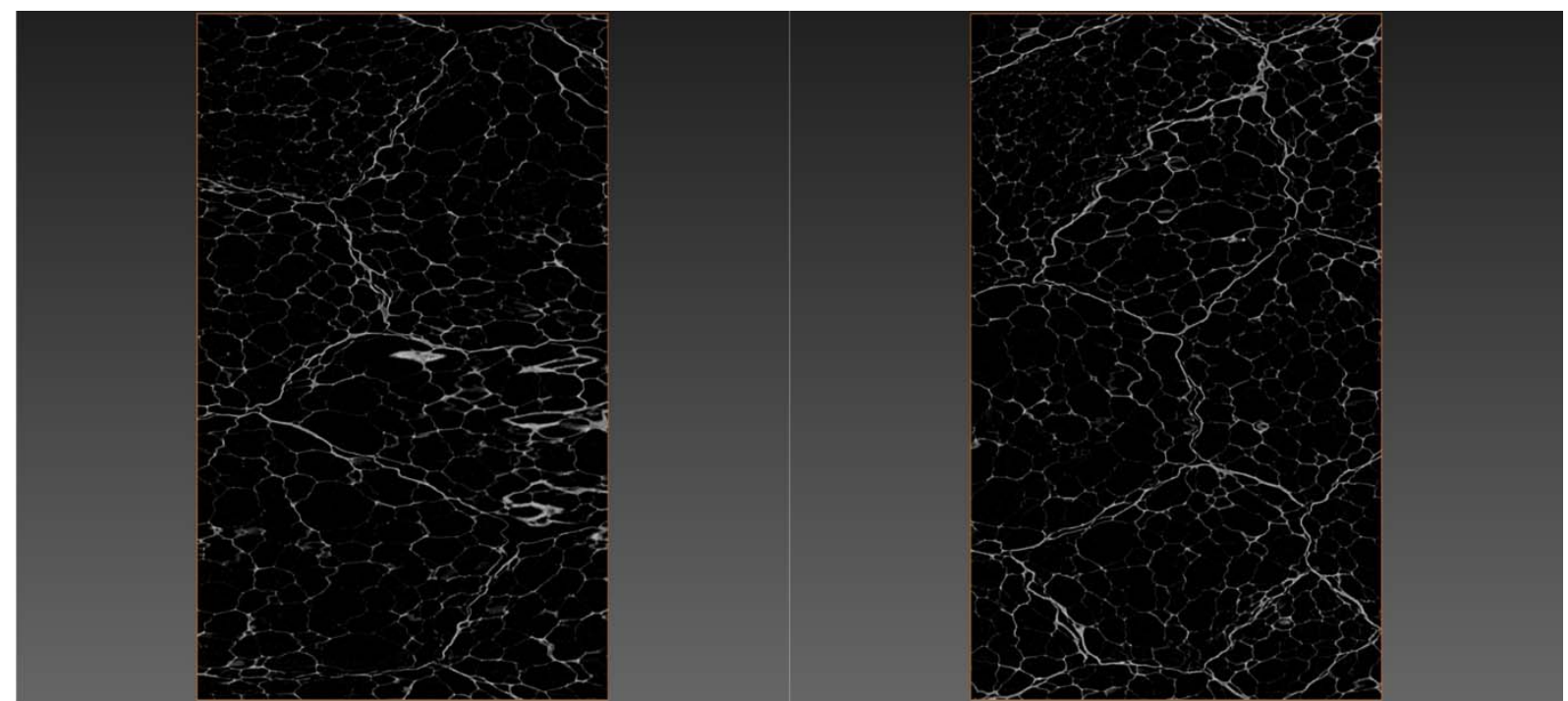

Fig. 15 Two vertical orthogonal slices of the central part of the EPP-90 T sample, compressed along the vertical direction.

A comparison with Fig. 11 clearly shows the effects of the sample compression, with visible permanent cell deformation and wall buckling. A closer comparison between the shape of the cells of the tested and untested samples, can be made by observing Fig. 16 that shows the volume rendering of the largest two hundred voids: compressed cells display a more irregular shape and are clearly crushed. 


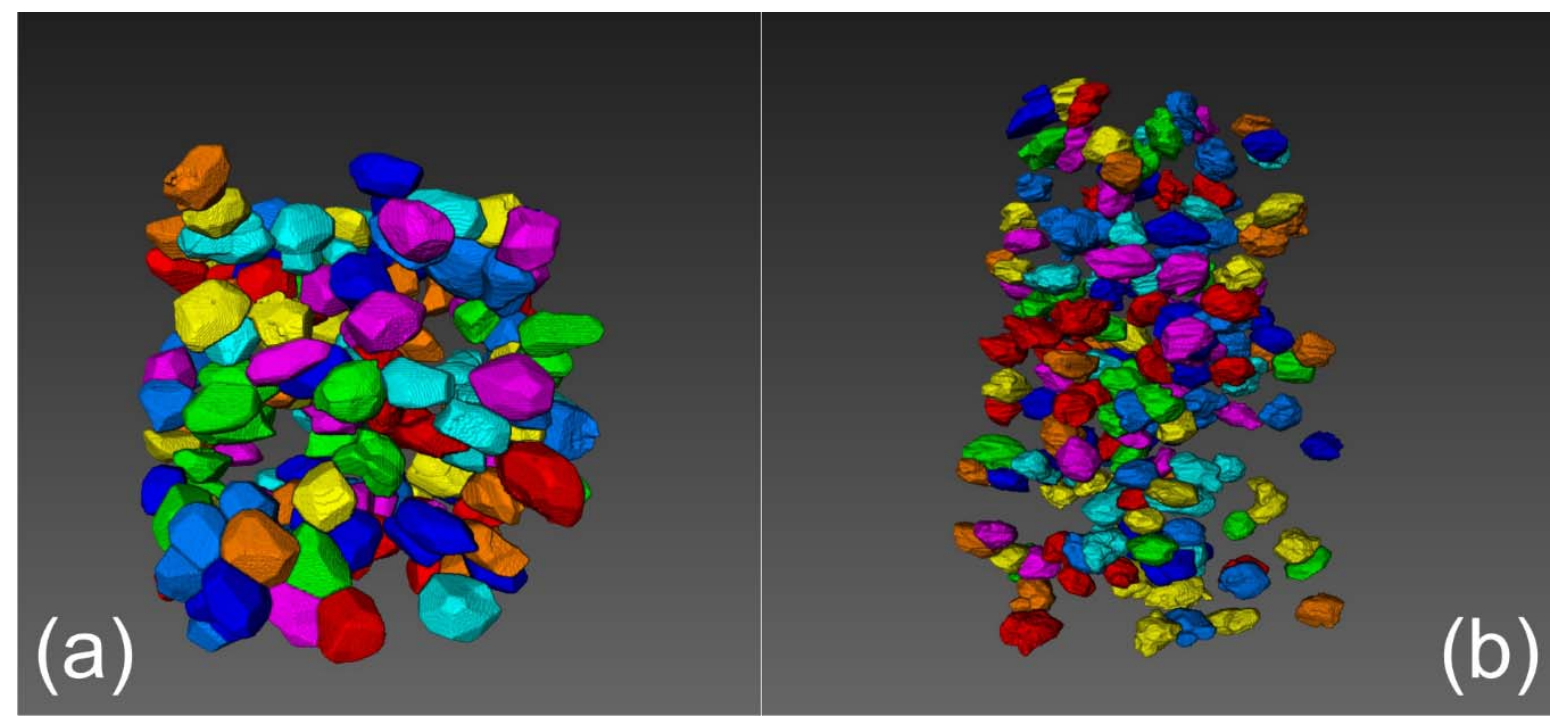

Fig. 16 Volume rendering of the largest 200 cells of EPP-90 foam: untested sample (a); tested sample (b).

Finally, the obtained analysis results are summarised in the graphs shown in Fig. 17, both for tested and untested EPP foam samples. It is quite clear that compression of the samples does not bring important changes on the evaluated parameters, except for sphericity which significantly decreases for three out of four densities considered.
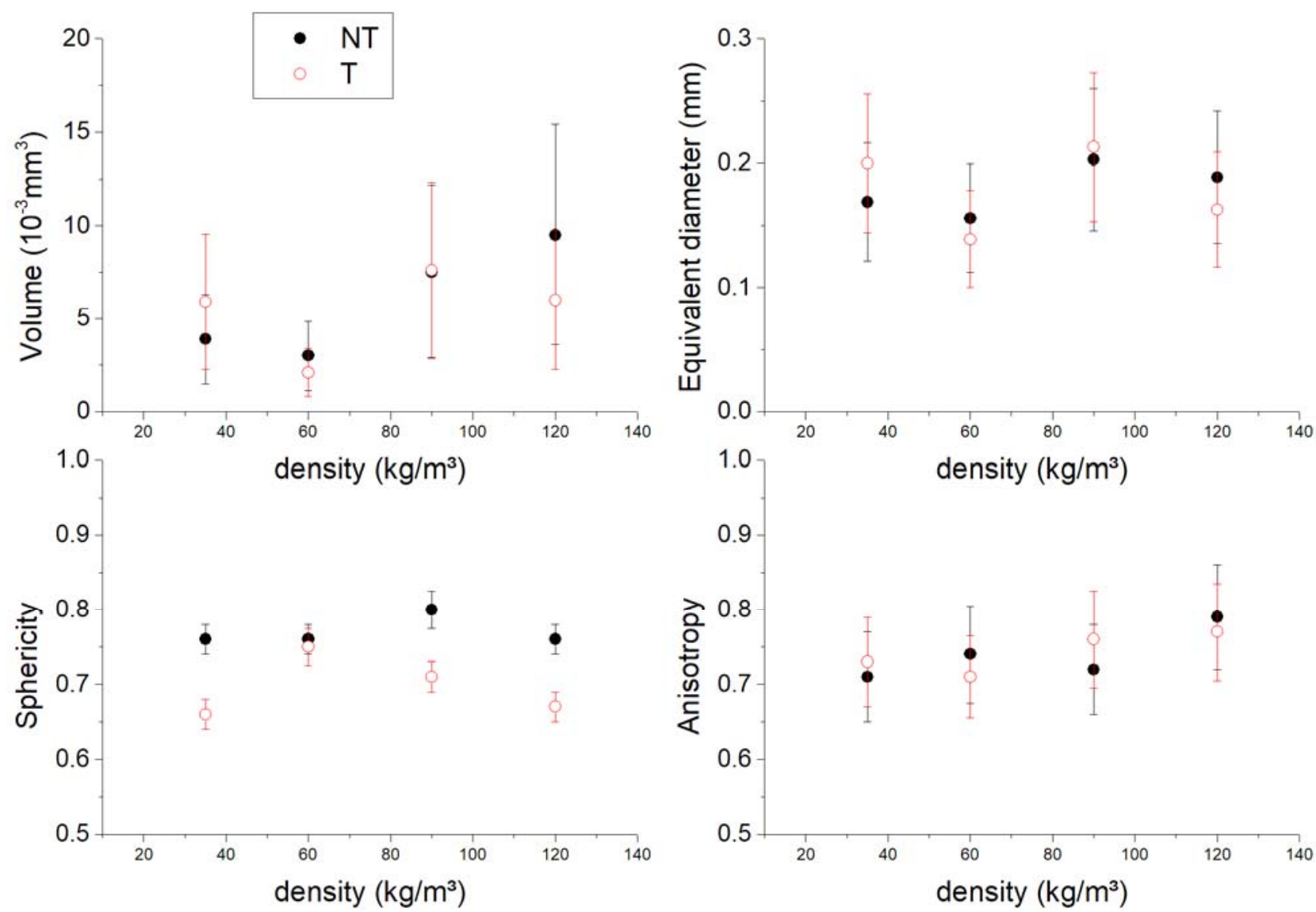

Fig. 17 Foam cells parameters obtained by $\mu-\mathrm{CT}$ analysis of untested (NT) and tested (T) EPP foam samples. Average values are reported with error bars indicating $95 \%$ confidence limits.

Fortunately, the results of the statistical analysis are not very sensitive to the choice of the threshold value for the filtering operation. If this value is varied in an acceptable range - e.g. from 0.60 to 0.66 for EPP-90 NT sample -, the observed changes affect at most the third significant digit. The resolution of SEM images at high magnification is of 
course better than that achievable in $\mu \mathrm{CT}$ reconstructed images and, for this reason, reliable information on cell wall thickness can be obtained only via SEM. To confirm this, an estimate of the apparent foam density was evaluated by calculating the volume fraction of the solid phase from the 3D reconstruction, obtaining values higher than the nominal ones: apparent density ranges from 1.3 times to 3.9 times the nominal density, for EPP-120 and EPP-35 samples, respectively. Where the number of cells per unit volume is greater and the wall thickness is smaller, the estimated value of the density differs more from the expected one. Based on these considerations, an attempt was made to calculate the average thickness of material in EPP-120 foam using tomographic data [25, 30]. The result of the computation was 9.8 $\mu \mathrm{m}$, as reported in Fig. 10(f). This average includes also contributions from beads' walls. The result is in fair agreement with that obtained with SEM, equal to 6.6 micron, especially if one takes into account that the spatial resolution of present tomographic images is $3.3 \mu \mathrm{m}$. On the other hand, $\mu \mathrm{CT}$ enables to perform analysis by penetrating deep into the sample and directly measuring 3D features, which could otherwise only be inferred from 2D images.

Thanks to $\mu \mathrm{CT}$, statistical evaluation of a very large number of cells included in the VOI of a typical analysis becomes possible. Yet, the large dispersion of the cell size population can limit significance of the analyses performed on selected regions of single samples. Future studies on these materials will have to consider at least a few samples per material/condition and/or in-situ testing. The latter would allow to establish a consistent correlation between a specific sample's microstructure and measured mechanical properties.

The very fine microstructure of the investigated EPP materials already pushes the resolution capabilities of the $\mu$ CT scanner presently used to their limits. Preliminary attempts on EPS samples demonstrated that with currently available means it is only possible to identify bead boundaries in a reliable way. Indeed, the estimated relative attenuation of the X-ray beam through $1 \mathrm{~cm}$ path in an EPS foam having a density of $25 \mathrm{~kg} / \mathrm{m}^{3}$ is less than $1 \%$. As a consequence, the image contrast is too weak to resolve cell walls; on the other hand, the thickness of the bead boundaries is larger than that of cell walls and can be observed. This information can still be quite useful since the bead meso-structure, which can be modified by processing parameters, is known to influence the performance of very low density foams used in the packaging sector. To the authors' knowledge, only a coherent X-ray source characterized by very high brightness (such as synchrotron radiation) can at present give the possibility of studying in detail these very low density polymeric foams.

The existing setup proved itself good enough to work on the relatively higher density foams, although the finerstructure regions close to the bead walls had to be excluded from the analysis. A potential limitation of this choice lies in its arbitrariness, should these regions (whose extension is actually quite limited) significantly affect the overall mechanical response. Modelling could help in investigating this issue, as numerical simulations could provide some guidance in evaluating the influence of bead boundaries on relevant foam behaviour.

\section{Conclusions}

In the present work, an experimental investigation of the compression behaviour of two families of polymeric foams was conducted. In particular, expanded polypropylene (EPP) and polystyrene (EPS) having densities ranging between $35-120 \mathrm{~kg} / \mathrm{m}^{3}$ and $10-25 \mathrm{~kg} / \mathrm{m}^{3}$, respectively, were considered. The research goal was to generate relevant experimental data, compare them with existing models in the literature and examine the material microstructure, seeking for a correlation with the measured mechanical properties.

It was found that the well-known Gibson-Ashby model can provide a reasonably good description of the dependence of both elastic modulus and plateau stress for most of the foams under study. A notable exception lies in the $75 \mathrm{~kg} / \mathrm{m}^{3} \mathrm{EPP}$ foam, which deviates from the common trend with density exhibited by the other EPP foams; moreover, this material shows a certain degree of mechanical anisotropy. These differences arise from a distinct microstructure which was reported by SEM and $\mu \mathrm{CT}$ analyses. This result highlights the influence that foam microstructure can have on the final properties. Modifications to the production process can generate effects that cannot be predicted based on density alone and microstructure can be optimized to improve foam properties for a given application. In this respect, modelling of the actual microstructural features of an existing foam (as made possible by $\mu \mathrm{CT}$ data) seems a very promising approach to further investigate these aspects. On the other hand, the examined foams had a highly polydisperse cell population and a comparison between the microstructure of untested and tested samples should be performed on the same sample in order to be fruitful; for this reason, it would be very useful to carry out in-situ micro-CT analysis of tested samples [31]. 
In view of using experimental data for modelling purposes, the strain rate dependence of the compressive properties was also investigated. Results show that, at least in the range of strain rates considered, the very simple Nagy's phenomenological model can well describe the data.

Finally, Maiti's energy absorption diagrams were obtained for both families of foam, allowing a selection of the optimal density based on the specific need of a given application.

\section{Acknowledgments}

This work was supported by Fondazione Cariplo, Milano (Italy) under the project "Safer Helmets". The authors also recognize the support of the Advanced Manufacturing Lab (AMALA) of Politecnico di Milano.

\section{Declaration of Conflicting Interests}

The authors declare that there is no conflict of interest.

\section{References}

1. Zhai W, Kim Y, Jung DW, et al. Steam-Chest Molding of Expanded Polypropylene Foams. 2. Mechanism of Interbead Bonding. Ind Eng Chem Res 2011; 50:5523-5531.

2. Gibson LJ and Ashby MF. Cellular Solids Structure and Properties. 2nd Edition Cambridge (UK): Cambridge University Press, 1991.

3. Rusch KC. Energy-absorbing characteristics of foamed polymers. J Appl Polym Sci 1970; 14:1433-1447.

4. Rusch KC. Load-compression behavior of brittle foams. J Appl Polym Sci 1970; 14:1263-1276.

5. Burgess G. Consolidation of cushion curves. Packag Technol Sci 1990; 3:189-194.

6. Castiglioni A, Castellani L, Cuder G, et al. Colloids Surf A. Epub ahead of print 23 March 2017. DOI: . 10.1016/j.colsurfa.2017.03.049

7. Maiti SK, Gibson LJ and Ashby MF. Deformation and energy absorption diagrams for cellular solids. Acta Metal 1984; 32:1963-1975.

8. Srivastava V and Srivastava R.On the polymeric foams: modeling and properties. J Mater Sci 2014; 49:26812692.

9. Andena L, Caimmi F, Leonardi L, et al. Towards safer helmets: characterisation, modelling and monitoring. Proc Eng 2016; 147:478-483.

10. Thomson WS. On the division of space with minimum partitional area. Acta Math 1887; 11:121-134.

11. Shulmeister V. Modelling of the mechanical properties of low-density foams. PhD Dissertation, Delft University of Technology, NL, 1998.

12. Roberts AR and Garboczi EJ. Elastic moduli of model random 3-D closed-cell cellular solids. Acta Mater 2001; 49:189-197.

13. Wismans JGF, Van Dommelen JAW, Govaert LE, et al. Computed Tomography-based Modeling of Structured Polymers. J Cell Plast 2009; 45:157-179.

14. Andena L, Briatico F, Benanti M, et al. Characterization and modelling of the mechanical and acoustical properties of a PVC rigid foam. In: EUROTEC 2013, Lyon, France, 4-5 July 2013.

15. Fedele R, Ciani A, Galantucci L, et al. A regularized, pyramidal multi-grid approach to global 3D-Volume Digital Image Correlation based on X-ray micro-tomography. Fundamenta Informaticae 2013; 125:361-376.

16. Apostol D and Constantinescu D. Temperature and speed of testing influence on the densification and recovery of polyurethane foams. Mech Time-Depend Mater 2013; 17:111-136.

17. Avalle M, Belingardi G and Montanini R. Characterization of polymeric structural foams under compressive impact loading by means of energy- absorption diagram. Int J Impact Eng 2001; 25:455-472.

18. Mills NJ and Mills NJ. Deformation mechanisms and the yield surface of low-density, closed-cell polymer foams. J Mater Sci 2010; 45:5831-5843.

19. Viot P, Beani F and Lataillade J. Polymeric foam behavior under dynamic compressive loading. J Mater Sci 2005; 40:5829-5837.

20. Zenkert D and Burman M. Tension, compression and shear fatigue of a closed cell polymer foam. Compos Sci Technol 2009; 69:785-792.

21. Ouellet S, Cronin D and Worswick M. Compressive response of polymeric foams under quasi-static, medium and high strain rate conditions. Polym Test 2006; 25:731-743.

22. Nagy A, Ko WL and Lindholm US. Mechanical Behavior of Foamed Materials under Dynamic Compression. J Cell Plast 1974; 10:127-134. 
23. Scarpa F, Ciffo LG and Yates JR. Dynamic properties of high structural integrity auxetic open cell foam. Smart Mater Struct 2004; 13:49-56.

24. Zhang J, Kikuchi N, Li V, et al. Constitutive modeling of polymeric foam material subjected to dynamic crash loading. Int J Impact Eng 1998; 21:369-386.

25. Osher J and Schladitz K. 3D Images of Materials Structures: Processing and Analysis. Weinhem: Wiley-VCH, 2009.

26. De Pascalis F, Nacucchi M, Scatto M, et al. Quantitative characterisation of low-density,high performance polymeric foams using high resolution X-ray computed tomography and laser confocal microscopy. Nondestr Test Eng Int 2016; 86:123-133.

27. Merkus HG. Particle Size Measurements: Fundamentals, Practice, Quality. 1st Edition Cambridge (UK): Springer Netherlands, 2009.

28. Clift R, Grace JR and Weber ME. Bubbles, drops and particles. New York : Academic Press, 1978.

29. Gonzalez R and Woods RE. Digital image processing. 2nd ed. Upper Saddle River, New Jersey: Prentice Hall, 2002.

30. Nacucchi M., De Pascalis F., Scatto M., Capodieci L. and Albertoni R. Structural analysis of advanced polymeric foams by means of high resolution X-ray computed tomography AIP Conference Proceedings, 1749 , 020009 (2016); doi: 10.1063/1.4954492.

31. Burteau A., N'Guyen F., Bartout J.D., Forest S., Bienvenu Y., Saberi S., Naumann D. Impact of material processing and deformation on cell morphology and mechanical behavior of polyurethane and nickel foams. Int J Solid Struct 2012; 49:2714-2732

32. Tagliabue S., Rossi E., Baino F., Vitale-Brovarone C., Gastaldi D., Vena P. Micro-CT based finite element models for elastic properties of glass-ceramic scaffolds. J Mech Behav Biom Mat 2017; 65:248-255

\section{Figure Captions}

Fig.1 Typical uniaxial compression stress-strain behaviour of a closed cell foam. The grey area represents the absorbed energy per unit volume.

Fig.2 Uniaxial compression stress-strain behaviour of EPP-75, EPP-90 and EPS-19 at a strain rate of 3.2 $10^{-2} \mathrm{~s}^{-1}$.

Fig.3 Effect of material density on the compression stress-strain behaviour of EPP (thickness direction) and EPS at a strain rate of $3.2 \cdot 10^{-3} \mathrm{~s}^{-1}$. Labels indicate foam density.

Fig.4 Effect of strain rate on the compression stress-strain behaviour of EPP-90 and EPS-19.

Fig.5 EPP and EPS elastic modulus data represented as a function of nominal density. Continuous lines: fit to Eq.2.

Fig.6 EPP and EPS plateau stress data (at 25\% compressive strain) represented as a function of nominal density.

Continuous lines: fit to Eq.1.

Fig.7 EPP and EPS plateau stress data (at 25\% compressive strain) represented as a function of strain rate. Continuous lines: fit to Eq.3. Labels indicate foam density.

Fig.8 Dependence of the strain rate index $n$ (see Eq. 3) on the compressive strain for EPP and EPS.

Lines represent a linear fit of the data.

Fig. 9 Energy absorption diagram for EPS and EPP foams. Different colors correspond to different densities; see the online version of this figure. Labels below the data points refer to the EPP foams, while labels above refer to the EPS ones.

Fig. 10 EPP foam microstructure: SEM images for selected densities ((a)-(c)). Diagrams of mean Feret diameter (d), cell area (e) and wall thickness (f) distribution; dashed lines represent a linear fit of all data except for EPP-75 (in red). Error bars indicate the standard deviation of the measurements. For Feret diameter and wall thickness, available $\mu \mathrm{CT}$ data are also included.

Fig. 11 Orthogonal slices of the central part of the EPP-90 NT sample. (a) virtual section along the thickness direction; (b) virtual section along the perpendicular plane (in-plane).

Fig. 12 The same orthogonal slices of the central part of the EPP-90 NT sample as in Fig. 11(a-b), after the segmentation process. The border cells were removed.

Fig. 13 Volume rendering of the 100 largest rejected cells, after the filtering process. Note the flattened shape of the cells.

Fig. 14 Histograms of equivalent diameter (a) and anisotropy (b) distributions related to foam EPP-90 NT. 
Fig. 15 Two vertical orthogonal slices of the central part of the EPP-90 T sample, compressed along the vertical direction.

Fig. 16 Volume rendering of the largest 200 cells of EPP-90 foam: untested sample (a); tested sample (b).

Fig. 17 Foam cells parameters obtained by $\mu$-CT analysis of untested (NT) and tested (T) EPP foam samples. Average values are reported with error bars indicating $95 \%$ confidence limits. 